Further section

Int. Arch. Allergy 1973;44:736

\title{
Obituary Note
}

Professor Werner Jadassohn, former director of the Dermatological Clinic of the University of Geneve, member of the Collegium Internationale Allergologicum, died on June 5, 1973. 